\title{
Correlation Between Orthostatic Hypotension, Cognition, and Balance Control Symptoms of Parkinson's Disease with the Level of Vitamin D
}

\author{
Tingjia Shi ${ }^{1, *}$ \\ ${ }^{1}$ University of Pittsburgh; Biological Science; Pittsburgh, PA, 15213, United States \\ *Corresponding author's e-mail: ShiLiShuang@cas-harbour.org
}

\begin{abstract}
Parkinson's Disease is a nowadays common disease in the daily life. Many people with this disease often display the motor and non-motor symptoms. Existing research suggested that some symptoms related to the level of vitamin D concentration. However, whether there is a relationship between them still lacks a conclusion. Thus, through analyzing and comparing the research of Jang, Wang, and Peterson, the author aimed to prove that the level of vitamin D concentration in senior people with Parkinson's Disease correlated with orthostatic hypotension, cognition, and balance control symptoms.

According to the analysis of these studies, the first experiment showed the levels of 25 -hydroxyvitamin $\mathrm{D}$ and calcitriol were reduced in PD patients with orthostatic hypotension compare to without orthostatic hypotension. The second experiment reported the PD severity and serum vitamin D levels were inversely associated. Lastly, the higher vitamin D concentration, the better neuropsychiatric performance in the non-demented subset. Therefore, the correlation between vitamin $\mathrm{D}$ with orthostatic hypotension, mood and cognition, as well as balance control was proved to be existed.
\end{abstract}

Keywords: Vitamin D; Parkinson's Disease, Neurodegeneration, Neuroprotection, Orthostatic Hypotension, Mood, Cognition, Balance Control Disorder

\section{INTRODUCTION}

Over 10 million people detected Parkinson's Disease (in the following paper, PD will be referred to Parkinson's Disease) in the world and suffering with motor and non-motor symptoms that are commonly found in $\mathrm{PD}$, which are characterized by postural instability, orthostatic hypotension (in the following paper, $\mathrm{OH}$ will be referred to orthostatic hypotension), cognitive impairment, and dementia, etc. Vitamins D2 and D3 are the most important members of vitamins D that belongs to the fat-soluble vitamins. The former is produced by ultraviolet irradiation of subcutaneous 7dehydrocholesterol, while the latter is produced by ultraviolet irradiation of ergosterol, which is found in plants or yeast [5]. The traditional belief is that the main function of vitamin D is to promote cell growth, differentiation and regulation of immune function, in order to promote the absorption of calcium and phosphorus in the mucosal cells of the small intestine. In recent decades, research has found that vitamin $\mathrm{D}$ greatly affects the development of brain nerves and the regulation of neural activity [14]. Several studies have examined the asscoiation between vitamin $\mathrm{D}$ with motor and non-motor symptoms of PD. The pathophysiology of $\mathrm{OH}$ in $\mathrm{PD}$ is a neurodegenerative process. The level of vitamin D may significantly relate to $\mathrm{OH}$ in $\mathrm{PD}$ [1]. Neuropsychiatric manifestations such as cognition and mood in PD patients will become severe or moderated with vitamin D levels [2]. Motility will also be diminished by neurodegenerative process [3]. Therefore, a literature review will first clarify how level of vitamin $\mathrm{D} 3$ associated with $\mathrm{OH}$ and how it affected the risk of $\mathrm{PD}$, then explain the association between vitamin $\mathrm{D}$ and neuropsychological dysfunction. Lastly, how vitamin D influence balance function of PD patients. This study aims to provide implications of PD treatment in humans for the future research. 


\section{THE INFLUENCE OF VITAMIN D ON OH OF PD PATIENTS}

$\mathrm{OH}$ is one of the non-motor symptoms in PD that determined by a decrease in systolic blood pressure (BP) of $\geq 20 \mathrm{~mm} \mathrm{Hg}$ or in diastolic BP of $\geq 10 \mathrm{~mm} \mathrm{Hg}$, when the position changed from supine or sitting to stand up within 3 minutes [4]. The patients may experience symptoms such as dizziness, blurry vision, and discomfort with head or neck. Studies have indicated that vitamin $\mathrm{D}$ in neuroprotective may associated with $\mathrm{OH}$, since $\mathrm{OH}$ has been considered as neurodegenerative process. In particular, McCarroll et al. suggested that vitamin $\mathrm{D}$ levels were lower in patients with $\mathrm{OH}$ which indicated vitamin D may play an aetiological role [7]. However, the specific mechanism for this association remains unanswered.

An observational study leading by Jang et al. investigated 55 patients with PD and tested their blood pressure while the active standing test, then divided the $\mathrm{PD}$ patients into two groups with and without $\mathrm{OH}$. The levels of serum 25-hydroxyvitamin D and calcitriol was compared between the two groups, which was relatively low in the group with $\mathrm{OH}$ symptom. Furthermore, to testify the association between serum vitamin $\mathrm{D}$ levels and $\mathrm{OH}$ severity in $\mathrm{PD}$, the levels of serum 25hydroxyvitamin $\mathrm{D}$ and calcitriol was compared to systolic and diastolic BP changes and symptom severity which showed significant negative correlation [1]. Although the mechanism of effect of vitamin D on BP is not clear, it can be inferred from the neuroprotective effect of vitamin D that high levels of vitamin D may prevent degeneration of brain tissue. In Jang's experiment, they also studied other variables that reflect the severity of $\mathrm{OH}$. They compared the two groups with and without $\mathrm{OH}$ to the disease severity and disease duration respectively, however, the result presented disease severity and disease duration were no significant differences between the two groups. Therefore, the results show vitamin D levels did not associate with reduced mobility and disease duration in early PD patients.

The same result was also confirmed in Wang et al.'s experiment. They assumed that the patients will have lower vitamin D levels with longer disease duration due to reduced mobility, then they tested the level of $25(\mathrm{OH}) \mathrm{D} 2$ and $25(\mathrm{OH}) \mathrm{D} 3$ of the patients who have longer disease duration with reduced mobility. However, the results showed the disease duration have a small impact on the level of 25(OH)D3 and no correlation between the disease duration and 25(OH)D2, which means the reduction in mobility cannot explain the inverse relationship between disease duration and vitamin D concentration [5].

Accordingly, it has been suggested that Wang's result further explained Jang's experimental results that the severity and duration of the disease were similar within the two groups. The reduction of vitamin $\mathrm{D}$ in the $\mathrm{OH}$ group is unlikely due to increased disease severity and reduced mobility. Due to the small sample size in this study, the influence of vitamin on $\mathrm{OH}$ in $\mathrm{PD}$ remains unclear, but Jang's study provides useful information for a subsequent longitudinal study.

\section{THE INFLUENCE OF VITAMIN D ON THE NEUROPSYCHOLOGICAL SYMPTOMS OF PD PATIENTS}

Studies have confirmed that the deficiency of vitamin D can cause neuropsychological non-motor symptoms, such as anxiety, dementia, depression, and cognitive dysfunction, in PD patients. On the other hand, it is possible that vitamin D may also have a protective effect in neurodevelopment. But few experiments have been able to confirm the effect of vitamin D levels on cognition in PD [8][9].

To understand the relationship between vitamin D and neuropsychological function, a group of scientists led by Peterson conducted neuropsychological testing on 286 participants with PD. PD participants were divided into two groups, demented and non-demented, the Verbal Fluency analysis [10], Verbal Memory analysis [11], and Geriatric Depression Scale (GDS) [12] were performed simultaneously in both groups [2]. The verbal fluency and verbal memory analysis showed significant relationship between vitamin $\mathrm{D}$ concentration and cognitive function in the non-demented group based on the neuropsychological measurements in the multivariate model, while the demented group showed no significant correlation between the cognitive test and vitamin $\mathrm{D}$. The GDS was used for analyzing the correlation between levels of vitamin D and depression, which showed a significant relationship in non-demented group. Therefore, the results showed vitamin D is significantly associated with different neuropsychological performance of verbal fluency, verbal memory, and depression in non-demented group [2].

Depression is known to aggravate cognitive function. To further understand the relationship between vitamin $\mathrm{D}$ and cognitive function, Peterson et al. performed a mediation analysis due to GDS score. In cognitive measurements, the verbal fluency analysis was closely associated to vitamin D level in the non-demented group, whereas no significant connection between cognitive test and vitamin $\mathrm{D}$ is found in the demented group. In the view of mood measurements, a significant association between vitamin D levels and GDS was presented in the non-demented group. According to the mediation analysis, vitamin $\mathrm{D}$ has a direct effect on cognitive measurements but no major mediating effect on depression [2]. However, Peterson's results have suggested a potential benefits of vitamin D supplements 
to neuropsychological function (mood and cognitive), which also paved the way for future study.

\section{THE INFLUENCE OF VITAMIN D ON THE BALANCE FUNCTION OF PD PATIENTS}

Countless studies showed that balance and fall issue has always been a fatal issue for PD patients. Researcher Peterson's team introduced a cross-sectional and observational study to find the relationship between balance control and vitamin D in PD patients. The study focused on how PD patients reacted to their self-balance in motions and their balance control on external forces. The researchers examined the study in three different experiments: The Motor Control Test (MCT), The Sensory Organization Test (SOT), and The Sit to Stand test (STS) [3].

The MCT focused on how PD patients retain balance from external force with a backward perturbation, which is linked with the automatic postural response. PD patients were measured with the correlation between vitamin D with response strength, symmetry, and latency. However, only the response strength and the symmetry showed that there is a correlation with vitamin D concentration with a significant $p$-value. The SOT examined body sway under six different sensory conditions and measured the participant's sensitivity of reaction to the movement. The result showed that only one out of six conditions was correlated with vitamin D concentration. However, the researchers could not determine whether sway and vitamin D in PD patients possess a correlation since there were many other factors that could influence balance [3].

The level of vitamin D in PD patients is correlated with their symptom severity. The receptors and final converting enzymes in the brain shows that the vitamin $\mathrm{D}$ may directly function on the central nervous system, which can conclude that vitamin D has an effect on PD patients significantly. However, researcher Peterson's team was not able to determine whether vitamin D deficiency leads to PD or vitamin D deficiency is caused by PD [3].

The average age of the participants for Peterson's study was 67 with a standard deviation of 9.6. This characteristic raises a question: does age matter when exploring vitamin D level in PD patients. A study done by Hiller's team explored the effect of high dose vitamin D supplementation in PD patients. The researchers found that vitamin D supplementation has a potential of more effective on younger PD patients, and high doses of vitamin D supplementation would raise risks of fall and balance issue in elder PD patients [13]. Overall, Peterson's results indicated the relationship between vitamin $\mathrm{D}$ and disease severity but the cause and effect between vitamin D deficiency and PD needs further study.

\section{DISCUSSION}

The significant correlation between vitamin $\mathrm{D}$ with motor and non-motor symptoms of PD is demonstrated in all three experiments. Jang's experiment suggested a low vitamin $\mathrm{D}$ status is associated with $\mathrm{OH}$ in $\mathrm{PD}$ patients. The systolic and diastolic BPs and symptom severity showed a significant negative correlation in $\mathrm{OH}$ group, according to "reverse causation", which may suggest that high level of vitamin D as a neuroprotective role could prevent neurodegeneration diseases. Peterson's neuropsychological test suggested that higher level of vitamin D may have potential advantages on cognition and mood in non-dementia PD patients. Nevertheless, vitamin $\mathrm{D}$ deficiency may exacerbate cognitive decline during the progression of PD. An inverse relationship between vitamin D and the severity of PD symptoms was shown in Peterson's balance test. The results suggested vitamin D supplementation could improve muscle cell growth therefore diminishing the loss of muscle strength in PD patients. In addition, Peterson's research results may suggest that vitamin D status could affect motor symptoms of PD patients, which could also support Jang's experiment of the vitamin D may influence $\mathrm{OH}$ symptom in PD patients.

\section{CONCLUSION}

In conclusion, this review has demonstrated that vitamin $\mathrm{D}$ is correlated with $\mathrm{OH}$, Cognition, and Balance Control Symptoms in senior people with PD. Although Jang's experiment could not clearly explain the specific mechanism of influence of vitamin $\mathrm{D}$ on $\mathrm{OH}$ in $\mathrm{PD}$ patients, it has confirmed the association between vitamin $\mathrm{D}$ and $\mathrm{OH}$ in $\mathrm{PD}$ and provides additional information that reduced mobility is less likely the explanation for lower vitamin $\mathrm{D}$ in $\mathrm{OH}$ group. Peterson et al. has reported the vitamin D is crucial in mood and cognition as well as balance control. There are limitations to these studies, however, the research on the Multifunctionality of Vitamin D to treat and improve neurodegenerative diseases has opened up to the future studies. Appropriate vitamin D supplementation is effective in preventing and treating osteoporosis in elderly patients with PD, and may also have potential neuroprotective effects. However, there is no clear conclusion as to whether vitamin $\mathrm{D}$ deficiency contributes to the development of neurodegenerative diseases such as PD. Therefore, in the future, it is necessary to further confirm the current conclusion. In addition, exploring the effectiveness of vitamin D supplements for various disease symptoms and patients' life-quality needs to be carried out.

\section{ACKNOWLEDGMENT}

Thanks to Dr. Kelsey for her valuable and constructive suggestions of this literature review. 


\section{REFERENCES}

[1] W. Jang, J. Park, J.S. Kim, J. Youn, E. Oh, K.Y. Kwon, K.D. Jo, M.K. Lee, and H.T. Kim. 2015. Vitamin D deficiency in Parkinson's disease patients with orthostatic hypotension. Acta Neurol Scand. $132: 242-250$

[2] A.L. Peterson, C. Murchison, C. Zabetian, J.B. Leverenz, G.S. Watson, T. Montine, N. Carney, G.L. Bowman, K. Edwards, and J.F. Quinn. Memory, mood, and vitamin D in persons with Parkinson's disease. J Parkinsons Dis. 2013b, 3:547-555.

[3] A.L. Peterson, M. Mancini, and F.B. Horak. The relationship between balance control and vitamin $\mathrm{D}$ in Parkinson's disease-a pilot study. Mov Disord. 2013a, 28:1133-1137.

[4] R. Freeman, W. Wieling, F.B. Axelrod, D.G. Benditt, E. Benarroch, I. Biaggioni, W.P. Cheshire, T. Chelimsky, P. Cortelli, C.H. Gibbons, D.S. Goldstein, R. Hainsworth, M.J. Hilz, G. Jacob, H. Kaufmann, J. Jordan, L.A. Lipsitz, B.D. Levine, P.A. Low, C. Mathias, S.R. Raj, D. Robertson, P. Sandroni, I. Schatz, R. Schondorff, J.M. Stewart, and J.G. van Dijk. Consensus statement on the definition of orthostatic hypotension, neurally mediated syncope and the postural tachycardia syndrome. Clin Auton Res. 2011, 21:69-72.

[5] L. Wang, M.L. Evatt, L.G. Maldonado, W.R. Perry, J.C. Ritchie, G.W. Beecham, E.R. Martin, J.L. Haines, M.A. Pericak-Vance, J.M. Vance, and W.K. Scott. Vitamin D from different sources is inversely associated with Parkinson disease. Mov Disord. 2015, 30:560-566.

[6] M. Morello, V. Landel, E. Lacassagne, K. Baranger, C. Annweiler, F. Feron, and P. Millet. Vitamin D Improves Neurogenesis and Cognition in a Mouse Model of Alzheimer's Disease. Mol Neurobiol. 2018, 55:6463-6479.

[7] K.G. McCarroll, D.J. Robinson, A. Coughlan, M. Healy, R.A. Kenny, and C. Cunningham. Vitamin D and orthostatic hypotension. Age and Ageing. 2012, 41:810-813.

[8] C.A. Anastasiou, Yannakoulia M, Scarmeas N. Vitamin D and cognition: an update of the current evidence. J Alzheimers Dis. 2014;42 Suppl 3:S7180. doi: 10.3233/JAD-132636. PMID: 24820017.

[9] A.P. Dickens, I.A. Lang, K.M. Langa, K. Kos, D.J. Llewellyn. Vitamin D, cognitive dysfunction and dementia in older adults. CNS Drugs. 2011 Aug;25(8):629-39. doi: 10.2165/11593080000000000-00000. PMID: 21790207; PMCID: PMC5097668.
[10] M.D. Lezak, D.B. Howieson, D.W. Loring. Neuropsychological Assessment. 2004

[11] A.M. Shapiro, R.H. Benedict, D. Schretlen, J. Brandt. Construct and concurrent validity of the Hopkins Verbal Learning Test-revised. Clin Neuropsychol. 1999 Aug; 13(3):348-58.

[12] J. Sheikh, J. Yesavage. Clinical Gerontology: A Guide to Assessment and Intervention. Anonymous The Haywood Press; New York: 1986. Geriatric Depression Scale (GDS): Recent evidence and development of a shorter version; pp. $165-173$.

[13] A.L. Hiller, C.F. Murchison, B.M. Lobb, S. O'Connor, M. O'Connor, J.F. Quinn. A randomized, controlled pilot study of the effects of vitamin D supplementation on balance in Parkinson's disease: Does age matter? PLoS One. 2018 Sep 26;13(9):e0203637. doi: 10.1371/journal.pone.0203637. PMID: 30256811; PMCID: PMC6157857.

[14] I. Anjum, S.S. Jaffery, M. Fayyaz, Z. Samoo, S. Anjum. The Role of Vitamin D in Brain Health: A Mini Literature Review. Cureus. 2018 Jul 10;10(7):e2960. doi: 10.7759/cureus.2960. PMID: 30214848; PMCID: PMC6132681. 